\title{
The VIIRS Ocean Data Simulator Enhancements and Results
}

\author{
Wayne D. Robinson, ${ }^{a}$ Fredrick S. Patt, ${ }^{a}$,Bryan A. Franz ${ }^{b}$ \\ Kevin R. Turpie, ${ }^{a}$ and Charles R. McClain ${ }^{b}$ \\ ${ }^{a}$ Science Applications International Corporation, Beltsville, Maryland 20705, USA \\ ${ }^{b}$ NASA Goddard Space Flight Center, Greenbelt, Maryland 20771, USA
}

\begin{abstract}
The VIIRS Ocean Science Team (VOST) has been developing an Ocean Data Simulator to create realistic VIIRS SDR datasets based on MODIS water-leaving radiances. The simulator is helping to assess instrument performance and scientific processing algorithms. Several changes were made in the last two years to complete the simulator and broaden its usefulness. The simulator is now fully functional and includes all sensor characteristics measured during prelaunch testing, including electronic and optical crosstalk influences, polarization sensitivity, and relative spectral response. Also included is the simulation of cloud and land radiances to make more realistic data sets and to understand their important influence on nearby ocean color data. The atmospheric tables used in the processing, including aerosol and Rayleigh reflectance coefficients, have been modeled using VIIRS relative spectral responses. The capabilities of the simulator were expanded to work in an unaggregated sample mode and to produce scans with additional samples beyond the standard scan. These features improve the capability to realistically add artifacts which act upon individual instrument samples prior to aggregation and which may originate from beyond the actual scan boundaries. The simulator was expanded to simulate all $16 \mathrm{M}$-bands and the EDR processing was improved to use these bands to make an SST product. The simulator is being used to generate global VIIRS data from and in parallel with the MODIS Aqua data stream. Studies have been conducted using the simulator to investigate the impact of instrument artifacts. This paper discusses the simulator improvements and results from the artifact impact studies.
\end{abstract}

Keywords: NPP, VIIRS, ocean color, satellite remote sensing, simulator

\section{INTRODUCTION}

The VIIRS (Visible/Infrared Imaging Radiometer Suite) instrument scheduled to fly aboard the NPOESS Preparatory Project (NPP) satellite contains detectors in the visible and near-infrared range that should have the capability to measure Top Of Atmosphere (TOA) radiances with sufficient accuracy to permit the computation, with suitable processing software, of ocean color quantities such as water-leaving radiances and derived quantities such as chlorophyll-a concentration. ${ }^{1,2}$ The success of this task depends on the ability to reliably process the large quantities of data that will be operationally produced and to understand and be tolerant to the effects on retrievals of intervening atmospheric, ocean surface, and instrument features. The VIIRS Ocean Science Team (VOST) is developing a VIIRS data simulation system to address these issues. The simulator will help to assess the performance of VIIRS instrument and science software for the generation of ocean color parameters.

Previously, ${ }^{3}$ the VIIRS simulation system was described and examples were given. Since then, most of the additional features have been added to the system. Large improvements include the addition of simulated land and cloud radiances and the simulation of many instrument optical and electronic artifacts. Structural changes in the simulator design were made to accommodate the production of the artifacts. The substantially modified and improved simulation system will be reviewed again here.

Further author information:

e-mail: Wayne.D.Robinson@nasa.gov, phone: 3012863883 
The simulator has been integrated into the Ocean Biology Processing Group's (OBPG) Ocean Data Processing System (ODPS) to take advantage of its ability to direct large amounts of computer resources toward the processing of moderate resolution (about $1 \mathrm{~km}$ ) global daily datasets. In addition, the simulator software design takes advantage of the existing processing software used for the processing of other ocean color instrument data such as SeaWiFS on OrbView-2 and MODIS on Aqua and Terra. This will have 2 advantages: 1 - the simulator requires less new development due to the use of existing functions, and 2 - the processing shares a common algorithmic basis with other ocean color processing.

In this paper, the design of the VIIRS data simulator is discussed. The overall flow of the simulator will be shown followed by a more detailed discussion of the elements of the simulator. Examples of the input data and the resulting simulated VIIRS radiances will be shown and an initial analysis of the artifact influences on VIIRS products will be examined.

\section{OVERALL DESIGN OF THE VIIRS SIMULATOR}

The processing of NPP data will be performed by the Interface Data Processing Segment (IDPS) at the National Environmental Satellite, Data, and Information Service (NESDIS). VIIRS ocean data processing consists of a sequence of steps that process the raw satellite data into useful scientific quantities. First, the satellite data is placed in a Raw Data Record (RDR) format - which contains the instrument packet data. Radiances in raw counts make up the dataset at this point. The next level is the Sensor Data Record (SDR) that has the counts converted into actual radiance at the TOA. This data is then processed into Environmental Data Records (EDR), containing the data of interest to the ocean scientists such as normalized water-leaving radiances and chlorophyll-a. EDR data can then be mapped to a common projection, allowing easier time series comparison and averaging over longer time periods.

Figure 1 shows the major elements of the VIIRS simulator. Currently, the simulator creates VIIRS Sensor Data Records (SDR) for the moderate resolution (M) bands used for ocean color processing (the Visible and Near IR bands). The SDR contains the calibrated TOA radiances from the VIIRS instrument, navigation information, data quality information and identifying metadata. The simulator starts with a realistic VIIRS orbit description, a source of realistic water-leaving radiances and aerosol characteristics for the ocean color simulation, and surface reflectance information to be used for the land and cloud simulation. The steps in the simulation process are numbered (in Fig. 1) in the order in which they are performed in the course of a normal simulation. The steps are:

1. VIIRS geolocation and viewing geometry creation. Use a simulated orbit to generate the VIIRS-viewed geometry, including earth latitude and longitude, solar and satellite zenith and azimuth angles for all those points. The information is generated for the standard length VIIRS granule.

2. Create a VIIRS SDR without radiances. Create a simulated VIIRS SDR file based on the VIIRS-viewed geometry. The resulting file will be in the format of a VIIRS SDR file but only contains placeholder TOA radiances.

3. Ocean color and reflectance scene creation. Select ocean color satellite water-leaving radiance and atmosphere-corrected reflectance data to use in generating a special level-3 base scene containing all the required parameters to use in the simulation of the ocean, cloud, and land. The scenes should be compiled from at least 2 days of of data to achieve full geographic coverage.

4. Select ocean color and reflectance data at VIIRS locations. Extract parameters from the Level-3 base scenes and located at the VIIRS geographical locations. This makes a set of ocean surface radiances, reflectances and aerosol information as VIIRS would see them.

5. Propagate ocean radiances to TOA as seen by VIIRS. Re-construct the top of atmosphere radiances by running the OBPG level-2 processing program: 12gen, in the inverse mode. Atmospheric components seen with the VIIRS geometry are added back to the target file water-leaving radiances to make the TOA radiances. 


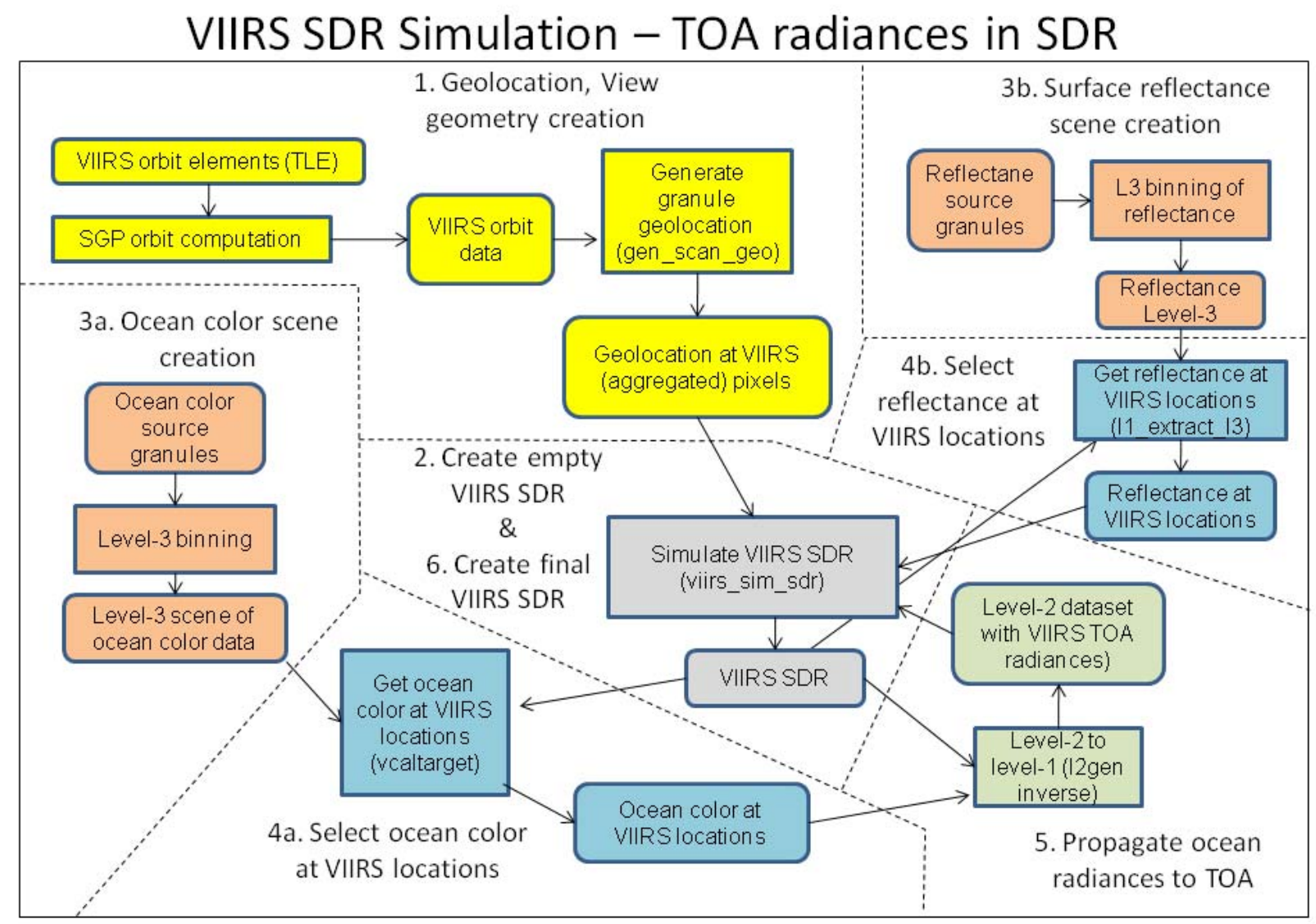

Figure 1. Flow chart depicting the major processing steps in the VIIRS data simulation. The numbers show the sequence of the simulation steps.

6. Simulate the VIIRS SDR with TOA radiances. Replace nominal TOA radiances in the VIIRS SDR file with either the ocean TOA radiances or the reflectances made into TOA radiances. Radiances are then modified by the instrument artifacts.

\section{DETAIL OF STEPS IN CREATING THE SIMULATED SDR}

The VIIRS simulation process takes a set of observed water-leaving radiances from the ocean surface combined with an estimate of the overlying atmosphere properties and simulates the radiances as seen by the VIIRS instrument at the top of the atmosphere. For non-ocean regions, a surface reflectance is converted to the TOA radiance.

Initially, the simulator used approximate tables of information about the VIIRS bands for testing purposes. The current version has tables for atmospheric correction and artifact addition that were derived from test measurements of the instrument characteristics under realistic environment conditions. Many of these quantities are listed in Table 1.

The simulation process is broken up into a set of steps described in the next sections. 
Table 1. VIIRS simulator information. The information, derived from measured instrument characteristics, in combination with other information and how it is used in the simulator

\begin{tabular}{|l|l|l|}
\hline Information & Derived from & Used for \\
\hline \hline Rayleigh radiance table & RSR and model & atmospheric correction \\
\hline Aerosol model radiances & RSR and model & atmospheric correction \\
\hline Band centers for the M bands & RSR & wavelength assignment \\
\hline Ozone absorption coefficients & RSR and model & atmospheric correction \\
\hline NO2 absorption coefficients & RSR and model & atmospheric correction \\
\hline $\begin{array}{l}\text { Extraterrestrial solar irradi- } \\
\text { ances }\end{array}$ & RSR and model & reflectance / radiance conversion \\
\hline CO2 transmittances & RSR and model & atmospheric correction \\
\hline H2O absorption coefficients & RSR and model & atmospheric correction \\
\hline Whitecap coefficients & RSR and model & atmospheric correction \\
\hline $\begin{array}{l}\text { Clear water absorption coeffi- } \\
\text { cients }\end{array}$ & RSR and model & atmospheric correction \\
\hline $\begin{array}{c}\text { Optical crosstalk influence coef- } \\
\text { ficients }\end{array}$ & Instrument tests & Optical crosstalk artifact \\
\hline Filter Spread functions & Instrument tests & Optical crosstalk artifact \\
\hline $\begin{array}{c}\text { Electronic crosstalk influence co- } \\
\text { efficients }\end{array}$ & Instrument tests & Electronic crosstalk artifact \\
\hline $\begin{array}{l}\text { Calibration gains, offsets } \\
\text { Blue ocean TOA interpolation }\end{array}$ & Modeling TOA radiance & $\begin{array}{l}\text { Band interpolation (for optical } \\
\text { crosstalk) }\end{array}$ \\
\hline $\begin{array}{l}\text { Reflectance vs. scan } \\
\text { coentsument tests }\end{array}$ & $\begin{array}{l}\text { Calibration / de-calibration (for } \\
\text { electronic crosstalk) }\end{array}$ \\
\hline
\end{tabular}

\subsection{VIIRS Geolocation and Viewing Geometry Creation}

The simulation begins with the creation of a set of location and view angle information for every pixel viewed by the VIIRS instrument (step 1 in Fig 1). A simulated orbit for NPP is generated from two-line element (TLE) sets, using the SGP4 program acquired from U.S. Project Spacetrack. The SGP4 model includes orbit perturbations to fourth order and also atmospheric drag. It allows orbit vectors to be output at any desired interval. For purposes of the VIIRS simulation, the vectors were generated at 1-minute intervals. The SGP4 vectors are in a true-equator, mean-equinox (TEME) reference frame. Prior to use in the simulation, they are transformed to the Earth-centered Earth-fixed (ECEF) frame. This involves rotating the vectors around the Earth's North Pole by the Greenwich Hour Angle (GHA), and also adjusting the velocity vectors for the Earth rotation rate. ${ }^{4}$

The NPP orbit is then used along with a nominal instrument attitude to derive the latitude and longitude of the aggregated VIIRS sample centers ${ }^{2}$ and the satellite and solar zenith and azimuth angles for these samples. The gen_scan_geo program interpolates the orbit information to the VIIRS scan rate of 1 scan per 1.7864 seconds. The sensor orientation is determined at each scan time using the orbit information and the nominal attitude. The VIIRS scan geometry, the sensor orientation, and an earth model are them used to determine the latitudes and longitudes of the scan pixels and the sensor zenith and azimuth angles. A reference sun vector, determined by the scan time, is used to determine the solar zenith and azimuth angles at the locations. The locations and view angles are placed in a temporary HDF-5 file. 
Table 2. VIIRS bands and the corresponding MODIS Aqua bands. Only the Vis and NIR bands are currently matched with Aqua

\begin{tabular}{|c|c||c|c|}
\hline VIIRS band & $\lambda$ (nm.) & Aqua band & $\lambda$ (nm.) \\
\hline \hline M1 & 410 & 8 & 412 \\
M2 & 443 & 9 & 443 \\
M3 & 486 & 10 & 488 \\
M4 & 551 & 12 & 551 \\
M5 & 671 & 13 & 667 \\
M6 & 745 & 15 & 748 \\
M7 & 862 & 16 & 869 \\
M8 & 1238 & & \\
M9 & 1378 & & \\
M10 & 1601 & & \\
M11 & 2257 & & \\
M12 & 3700 & & \\
M13 & 4050 & & \\
M14 & 8550 & & \\
M15 & 10763 & & \\
M16 & 12013 & & \\
\end{tabular}

The standard VIIRS granule can be created containing 48 (sometimes 47) scans which are made up of 16 lines (from the 16 detectors) and 3200 samples for each of the $16 \mathrm{M}$ bands. When the simulator is run with instrument artifacts included, the standard scan format is augmented to accommodate these needs. These additional formats are required because artifacts affect individual samples, before they are aggregated into the SDR values. Thus, the format can be in the un-aggregated mode - with each line containing 6304 samples instead of the standard 3200 (along with the proper geolocation and view information assigned to those samples). Some artifacts are derived using information from beyond the standard VIIRS scan. The optical and electronic crosstalk artifact is derived from 22 samples of information further along-scan. Stray light effects can depend on information in the along-track direction outside the standard 16 detector lines of a scan. For this reason, the simulated VIIRS scan can be augmented by additional samples along-scan and along-track. This non-standard scan format is carried through to the final simulation step, where it can then be trimmed and aggregated to the standard format if desired.

\subsection{Create a VIIRS SDR without Radiances}

This step (step 2 in Fig 1) uses the geolocation and view information to create a VIIRS SDR that conforms to the format specified in the NPOESS Common Data Format Control Book (CDFCB). ${ }^{5,6}$ The SDR is actually a set of files consisting of a geolocation file and a file for each of the 16 M-bands, including the 7 visible and near-IR bands used for ocean color processing (See the bands in Table 2). The VIIRS SDR dataset simulator program, viirs_sim_sdr, creates these datasets containing all the identifying metadata and quality information. The geolocation and view geometry are extracted from the geolocation file and are placed in the proper datasets. A temporary set of placeholder, band-dependent constant TOA radiances (or brightness temperatures for bands M12 - 16), based on typical TOA values, are placed in the appropriate M-band SDR file. The SDR is created in HDF 5 format as described by the CDFCB. 


\subsection{Ocean Color and Reflectance Scene Creation}

A scene of ocean and atmospheric properties at and above the ocean surface is generated using ocean color data from other instruments with similar wavelength bands. The current simulation processing uses water-leaving radiances from MODIS Aqua, which are at wavelengths very close to the visible and infrared wavelengths of VIIRS (see Table 2). Aerosol optical thickness and aerosol type- as indicated by the Angstrom coefficient are included to characterize the aerosol absorption properties in the atmosphere.

Individual Aqua scenes for a day do not fully cover the earth due to inter-orbit gaps. The ocean color data collection step (3a in Figure 1) takes the required parameters and accumulates them over a 2-day time period to attain full global coverage. Longer temporal averaging can also be done, but is not desirable as small features in the ocean would be smoothed out by the additional averaging. This step is performed using the available ODPS level-3 binning process: 13bin.

Similarly, surface reflectance data, from an instrument with suitably close, non-saturating bands close to the VIIRS wavelengths, is processed and binned globally over a 2-day time period ( $3 \mathrm{~b}$ in Figure 1 ). The source for the reflectance does not necessarily need to be from the same instrument, but in the case of the current simulation, the reflectance data is also derived from the MODIS Aqua instrument. The use of the same source instrument has the advantage that both the ocean color and reflectance data will be available over the same area at the same time. The Aqua land bands, which don't saturate over bright cloud and land areas, are chosen to make the reflectance datasets.

\subsection{Select Ocean Color and Reflectance Data at VIIRS Locations}

The simulated surface scenes, created in step 3 can now be sampled at the VIIRS sample locations. For the ocean color data, this step is done by the existing vcaltarget program (step 4a in Fig 1). vcaltarget was enhanced to use a VIIRS SDR as a source for location information and to access a less restrictive range of ocean radiance data. vcaltarget uses the latitude and longitude information from the VIIRS SDR to select the water-leaving radiances and aerosol information at those points in the surface scene. The information is also interpolated to the VIIRS wavelengths. Binary target files of this information are then created. vcaltarget can be adjusted to select only the Level-3 data that is in prescribed chlorophyll and aerosol optical thickness limits.

A separate process is used to select the reflectance data from the reflectance scene. The program 11_extract_13 selects reflectance from the Level-3 reflectance dataset and places it in the VIIRS SDR locations. A HDF-5 file of the selected reflectances is created.

\subsection{Propagate ocean radiances to TOA as seen by VIIRS}

The binary ocean data files created in step 4 are converted into TOA radiances in this step (step 5 in Fig 1). The standard OBPG Level-2 processing program, $12 \mathrm{gen}^{7}$ is used for this step. Normally, 12gen is used to remove the atmospheric influences from TOA radiances to create water-leaving radiances and aerosol properties. For the SDR simulation, 12gen is run in the inverse mode that takes the water-leaving radiances in the target file and adds atmospheric radiance contributions as seen by the VIIRS instrument for the view geometry specified in the SDR file. The inversion process uses the same software and principles as those used for the OBPG to perform the vicarious calibration of other ocean color instruments. ${ }^{8}$ The normalized waterleaving radiances are converted into water-leaving radiances as seen by the VIIRS instrument. The aerosol radiance, determined by the scene aerosol optical depth and angstrom coefficient is added to the waterleaving radiances ${ }^{9}$. Rayleigh radiances are then added and the effects of ozone absorption are accounted for. This results in a TOA radiance set for the ocean portions of the scene. The l2gen program places the TOA radiances in it's Level-2 format dataset. The existing 12 gen program was enhanced to work with the VIIRS SDR format data.

This step also obtains the atmospheric quantities required to convert the cloud and land reflectance data into TOA radiances for the non-ocean regions. 


\section{VIIRS Artifact addition detail}

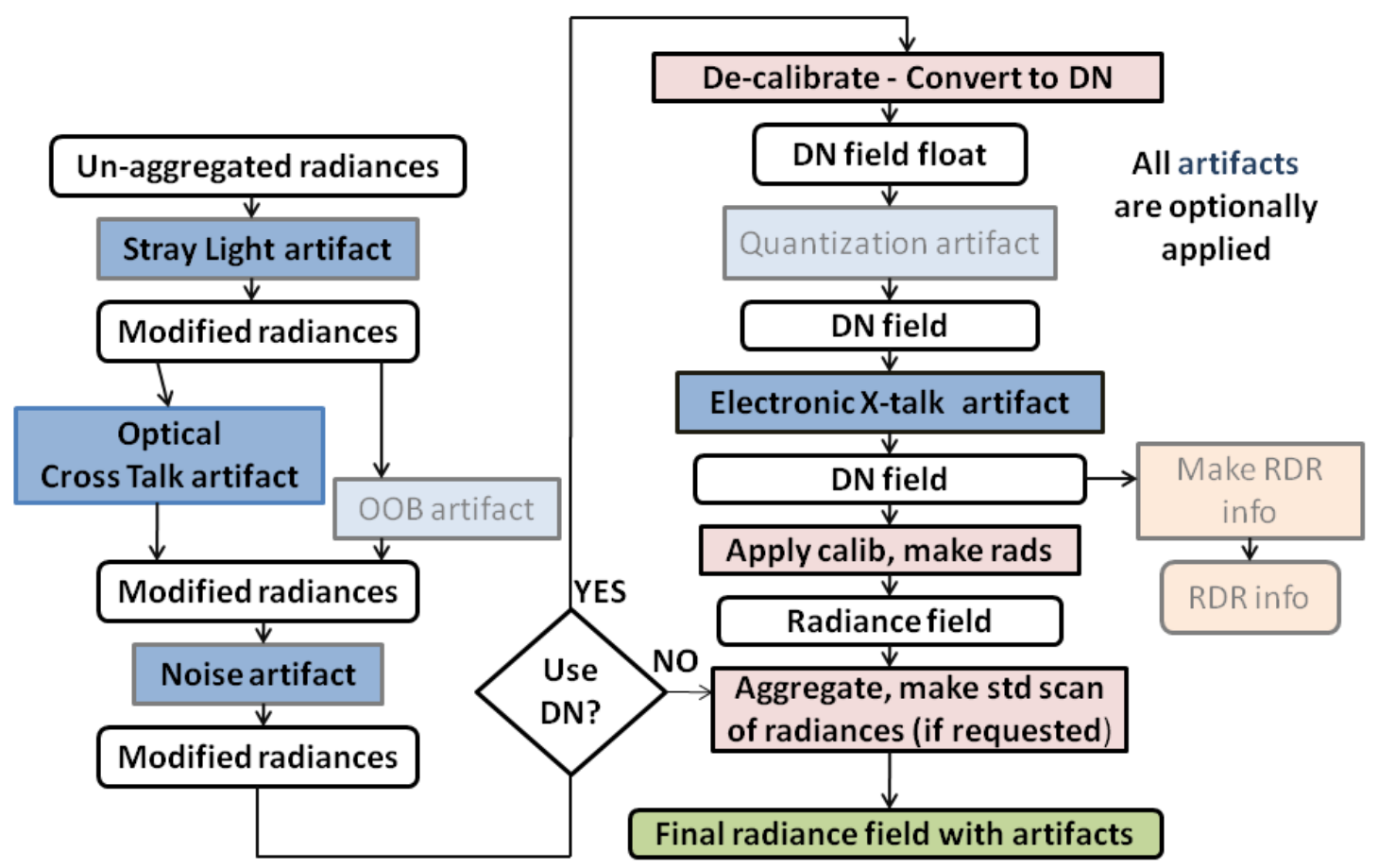

Figure 2. Flow chart showing the steps performed to add artifacts to the TOA radiances in the simulator step (Fig 1, step 6). The optical crosstalk, electronic crosstalk, stray light, and noise artifacts which are implemented are shown in dark color while the OOB and quantization artifacts, which are not yet implemented, are in half tone. Also, a possible future path to simulating the Raw Data Records (RDRs) is included.

\subsection{Simulate the VIIRS SDR with TOA radiances}

The last step of the simulation (step 6 in Fig 1) places the TOA radiances into the SDR file using the viirs_sim_sdr program. This step is similar to step 2, but in addition to inserting the geolocation information, the TOA ocean radiances are taken from the level-2 file and inserted into the simulated SDR. The reflectance data is also read, interpolated to VIIRS wavelengths, and converted from surface reflectance into TOA radiances, using the atmospheric properties over those areas. Normally, these radiances are used at sample locations where there is no ocean data available due to clouds, land or other conditions. An option exists to use only the ocean data or to use the reflectance data entirely. The visible and near-IR bands, M1-7 are treated in this way. Bands M8-16 are filled with typical TOA radiances for those bands. This is done so that the level-2 processing program (l2gen) can ingest all the M-bands as is expected in operational use. It also allows the VIIRS processing portion of 12 gen to handle the creation of SST products from the IR data. The processing of the simulated radiances is done on a scan-by-scan basis so that radiance values at any sample can be modified using the surrounding field of radiance values, which needs to be done when adding many of the simulated VIIRS instrument artifacts. Details of the VIIRS artifact addition is discussed in the next section.

\section{ARTIFACT SIMULATION DETAILS}

Many instrument artifacts can be added to the VIIRS radiances after the TOA radiances have been simulated (step 6 in Fig 1). The flow of the artifact addition in this portion of the processing is shown in Figure 2. 
The stray light, optical crosstalk, electronic crosstalk, and noise artifacts are currently implemented, but other artifacts are included in the flow in the order that they would be added to the TOA radiances.

Several instrument artifacts are included when the surface radiances are propagated to TOA (step 5 of Fig 1). These include the instrument polarization sensitivity, the relative spectral response of the bands (RSR) and the reflectance versus scan (RVS) influences. These are all corrections performed in the Level-2 or EDR creation.

The addition of certain instrument artifacts to the simulated VIIRS data requires the interpolation of TOA radiances to inter-band wavelengths, calibration and de-calibration of the radiances, and sample aggregation and scan trimming to convert the data into the standard VIIRS data format. Algorithms have been created to perform these steps.

The following sections describe these simulation elements.

\subsection{Stray Light}

Stray light, or near-field response is the radiance signal that spills over from its intended location and instead, falls on surrounding detectors. This effect is confined to the radiances in the wavelength of the detector and is solely a function of the separation from the source. Thermal vacuum tests derived some of the characteristics of the stray light and these measurements were combined with the responses for MODIS Aqua ${ }^{10}$ in the first few samples away from the bright target (BT) to derive the point spread function (PSF) for the VIIRS detectors for each band. Modifications were also made to convert the measurements made with illumination of all 16 detectors into an effect as if only one detector was illuminated. The PSF fields are generated for an along-scan range of 100 samples in both directions away from the central point of illumination and for all 16 detectors in the scan. Sets of these PSFs are made for all 7 VIS/NIR bands and for all the detectors in the scan.

The stray light artifact is applied by convolving the PSF with the radiances in the VIIRS scan. Although stray light effects will cross from one scan to the next, the test measurements limit the simulation to the along-track limits of a scan.

\subsection{Optical Crosstalk}

Optical crosstalk is the influence upon the radiance signal in one detector caused by radiance falling on another detector. It is distinct from electronic crosstalk in that it excludes any effects of the electrical signals from the detectors. Thermal vacuum tests (test FP-16) on the VIIRS focal plane determined a set of influence coefficients that give the change of radiance in a detector per unit of radiance seen in another band as a function of the illuminating wavelength. Other tests (ETP-655) determined a filter spread function for the influence of a detector upon another in the same band. These were combined to create a table of influence coefficients from every focal plane detector to every other detector's signal. The coefficients have several drawbacks: the influence coefficients have measurement errors associated with them, the measurements do not include polarization-dependent effects, imperfect illumination during testing required some coefficients to be zeroed out, and electronic effects may not have been completely removed from these coefficients. An application of these effects to a simulated dataset should give some idea of the impact of the actual artifacts on TOA radiances and derived Level-2 products, especially when comparisons can be made against the same data without artifacts added.

The application of the crosstalk artifact to the simulated radiances is done by convolving the radiance field seen by the detectors with the influence coefficients and adding the resultant influence radiance to that detector's radiance. Care must be taken to apply the coefficients to the correct sample to account for the physical position of the influencing band relative to the band being influenced. Also, the optical crosstalk is a function of not only the VIIRS radiances, but also of the radiances in wavelength ranges between the bands, from 370 to $1050 \mathrm{~nm}$ (the inter-band radiances). A method (described in section 4.8) was used to derive the inter-band radiances based on the TOA spectrum. 


\subsection{Electronic Crosstalk}

Electronic crosstalk is the change in a detector's signal arising from the detector electronics. The amount of influence depends on the strength of signals from all the other detectors and on the gain state of the sending and receiving detectors. A set of influence coefficients (in terms of receiver count change per count of sender) was derived with thermal vacuum test FP-13. These coefficients were made into a table for use during the simulation processing.

The application of the electronic crosstalk coefficients is done on the detector counts instead of the calibrated radiances. A de-calibration step must first be performed on the TOA radiances before the coefficients can be applied. This step yields both the count values required as well as the gain state used to convert measurement into counts (high gain for weak radiances and low gain for intense radiances). As with the optical crosstalk, the sample separation on the focal plane of the 2 bands must be accounted for so the correct sender count is used to get the influence on the receiver count. After the artifact is added to the counts, they are re-calibrated to produce radiances. The calibration and de-calibration is discussed in section 4.9 .

\subsection{Noise}

Noise is the random fluctuations of the voltage generated in the detectors and is proportional to the signal strength from the detectors. The signal to noise (SNR) of the radiances in the high gain setting was measured for the VIIRS bands in thermal vacuum tests. The SNR data, supplied by NICST, was analyzed to derive an SNR value as a quadratic function of the incident radiance for all bands. The coefficients of the quadratic fits are placed in a table for use in simulating the noise.

Application of the noise artifact to simulated VIIRS radiances involves deriving the SNR for each radiance, converting the SNR into a standard deviation for a Gaussian distribution, and then combining that with a random number generator output to derive the particular amount of noise which is added to that radiance sample. Over the course of many samples in a simulated dataset, the noise, or deviation of the radiance from the unaffected value, takes on a Gaussian distribution with the standard deviation consistent with the SNR at any particular radiance level.

\subsection{Polarization influences}

The optical train of the VIIRS instrument has some polarization-dependent responses. These sensitivities, when combined with the partially polarized components of the TOA radiances, such as the Rayleigh radiance influence the signal received by the VIIRS detectors and must be accounted for. The polarization response was measured in thermal vacuum testing ${ }^{11}$ and made into a table that is used to correct the Rayleigh radiances during the process that propagates surface radiances to TOA (Fig. 1, step 5). The testing of the sensitivity to these polarization effects can be investigated in the simulation by using one polarization table in the simulation and another when recovering the water-leaving radiances from the simulated data.

\subsection{Relative Spectral Response (RSR) influences}

The bands of the VIIRS instrument are primarily sensitive to a narrow range (about $20 \mathrm{~nm}$ ) of wavelengths. However, a significant portion of light in other wavelengths will also have an effect. This relative spectral response (RSR) of the VIIRS bands was measured during thermal vacuum testing and is used to compute the value of atmospheric components that go into the tables (Table 1) used to propagate the water-leaving radiances to TOA (Fig 1, step 5). It is also used to correct the water-leaving radiances derived from the TOA radiances so that they are more comparable to in-situ measurements.

\subsection{Reflectance versus scan (RVS) influences}

The signal at the VIIRS detectors varies as a function of the angle of incidence of incoming radiance on the scanning mirror and thus, position along scan. This RVS was determined during thermal vacuum testing and is included as a correction in the radiance calibration. The simulator applies a de-calibration and calibration step to the radiances in the process of adding the electronic crosstalk artifact. The cal, de-cal can also be done without adding the electronic artifact and it can be performed using different RVS tables during the calibration and de-calibration steps. This feature will make it possible to examine the sensitivity of the data to errors in the RVS. 


\subsection{Radiance Interpolation}

The optical crosstalk artifact computation requires values for the radiance in wavelength regions that are in-between those of the VIIRS bands. However, the simulation only has Aqua or SeaWiFS analogs (both surface radiances and atmospheric tables) for the similar VIIRS bands. This leaves the inter-band radiances to be determined.

A simple interpolation of the TOA radiances could be used to derive the inter-band values. For the simulator estimation, the simple interpolation was aided by a knowledge of the typical blue water TOA spectrum that has been derived by Barnes and Butler. ${ }^{12}$ The radiance average and radiance weighted center wavelength were determined for the 16 VIIRS in-band and inter-band wavelength ranges of the blue water TOA spectrum. Any radiance in a range can then be determined as a combination of nearby known radiances, weighted by the center wavelength separation and also modified by the radiance seen in the typical blue water spectrum. ${ }^{13}$ Once these inter-band radiances are calculated, the optical crosstalk coefficients can be applied.

\subsection{Calibration and De-calibration}

The electronic crosstalk artifact is computed from a the set of detector digital counts and the gain state they were taken in for all the visible and NIR bands. A de-calibration of the TOA radiances needs to be performed to obtain the count values. Likewise, following the crosstalk artifact addition, the counts need to be calibrated back into the TOA radiances. The VIIRS calibration ATBD ${ }^{14}$ describes the details of the calibration that was followed in the simulator. Since focal plane and other temperature measurements were not included in the simulation and for the current usage, the use of temperature dependent calibration corrections is not necessary, the calibration procedure used in the simulation was simplified to use gains and offsets determined from the instrument tests at a nominal temperature. For the de-calibration, it is initially assumed that each radiance is taken at the high gain state and the appropriate gain and offset are removed to get counts. For single gain bands, the conversion is complete. For dual gain bands, if the counts saturate the maximum count value for the band, the de-calibration is performed again using the low gain values of gain and offset. The count value and the gain setting are then ready for use in the electronic crosstalk calculation. The calibration can proceed using the proper gain and offset values for the radiance's gain state. It should be noted that the calibration step will produce not only the TOA radiance, but it will signal whether the radiance was saturated. Also, the count values created could be used to create a raw data record (RDR) dataset of the un-calibrated count values.

\subsection{Data aggregation and trimming}

When any instrument artifact is simulated, the TOA radiance samples need to be in the un-aggregated format to allow their proper application. Additionally, to allow the complete application of crosstalk artifacts at the end of scan, the standard scan must be extended by an additional 22 samples. The simulator is fitted with a controllable aggregating and trimming algorithm which can be applied to each scan after all artifacts are added. A program option can direct the simulator to leave the format unchanged, to trim any additional scan samples and lines, or to aggregate and trim the data. The data trimming is done as a simple subset. The aggregation needs to average samples in the active aggregation zones of the scan, excluding any flagged values. The saturation status of the aggregated sample needs to be set correctly and the geolocation and view angles for the aggregated samples need to be set as either the value of the central sample in the 3:1 aggregation zone or the angle mean value in the 2:1 aggregation zone (as done by converting the angles into vectors, computing the average vector and converting back to angles).

\subsection{Other instrument artifacts}

Figure 2 shows additional artifacts that could be added to the simulator in the future. These artifacts are:

1. Out of Band radiances (OOB). The OOB radiances are contributions to the signal in a detector from radiance on other detectors in the same band. 


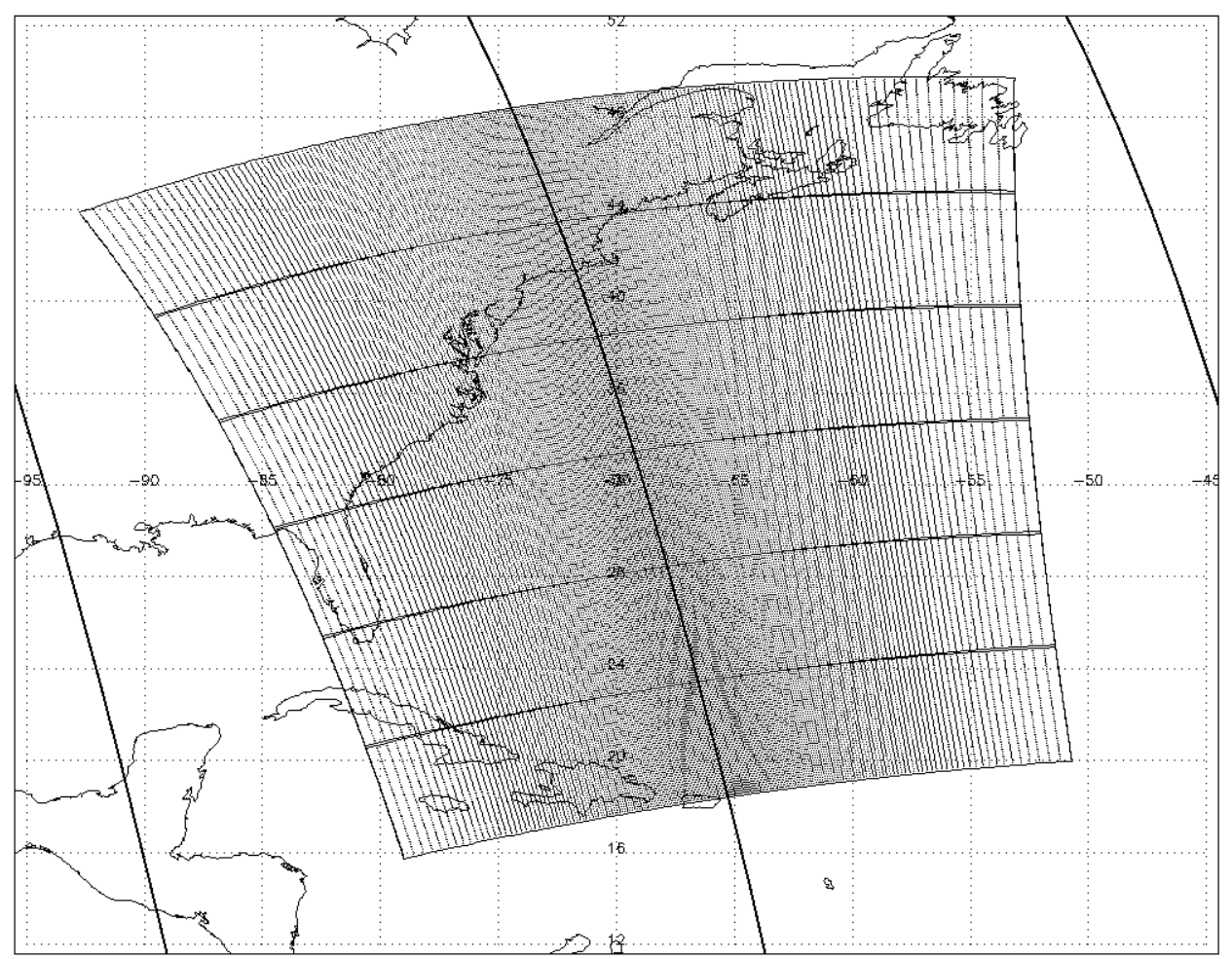

Figure 3. Plot of the VIIRS simulated granule coverage, using sub-sampled data point locations and the orbit ground track in the heavy black line.

2. Quantization. Quantization occurs when the analog voltage signal from a detector is converted into a digital count. The effects of quantization on an uncontaminated scene could be determined.

\section{SIMULATOR EXAMPLE}

An example of the simulator is discussed here and illustrated in Figures 3 to 6 . A region of the Atlantic Ocean surrounding the Bermuda Atlantic Time Series (BATS) station was chosen on a day when the station had relatively clear skies around it. The day was also chosen so that the simulated VIIRS orbit had BATS located near the center of the scan to simulate a good vicarious calibration event. The 13th of October, 2010 (day 286) was the date chosen for this example. Figure 3 shows the track of the NPP satellite subpoint over the region and the area covered by 6 granules chosen for the example. Selected sample locations from each granule were plotted in the figure. For purposes of clarity, only every 16th pixel along-scan is plotted. Also, only the first and last detector locations (out of the 16 detectors) have been plotted for the 48 scans that make up each granule. Note that since this example has artifacts added, the sample plot shows un-aggregated sample location spacing.

The MODIS Aqua data for the 12th and 13th of October were used to make the surface ocean and reflectance data. Figure 4 is the level-3 chlorophyll-a of the ocean data that was used as the basis for the simulation. The operational simulation uses 2-day composites of all the Aqua data over the entire globe.

The VIIRS simulated granules made from the Aqua data is shown as a true color image in Figure 5. Figure 6 is the chlorophyll-a image made from the level-2 dataset derived from the SDRs. The simulation results shown here include all the simulated artifacts of noise, optical and electronic crosstalk, and stray light. The true color image shows that the use of Aqua land band reflectance can create a very realistic image of land and cloud features. The simulated chlorophyll-a image (Fig 6) closely matches the chlor_a derived from the Aqua data used in the simulation (Fig 4). 


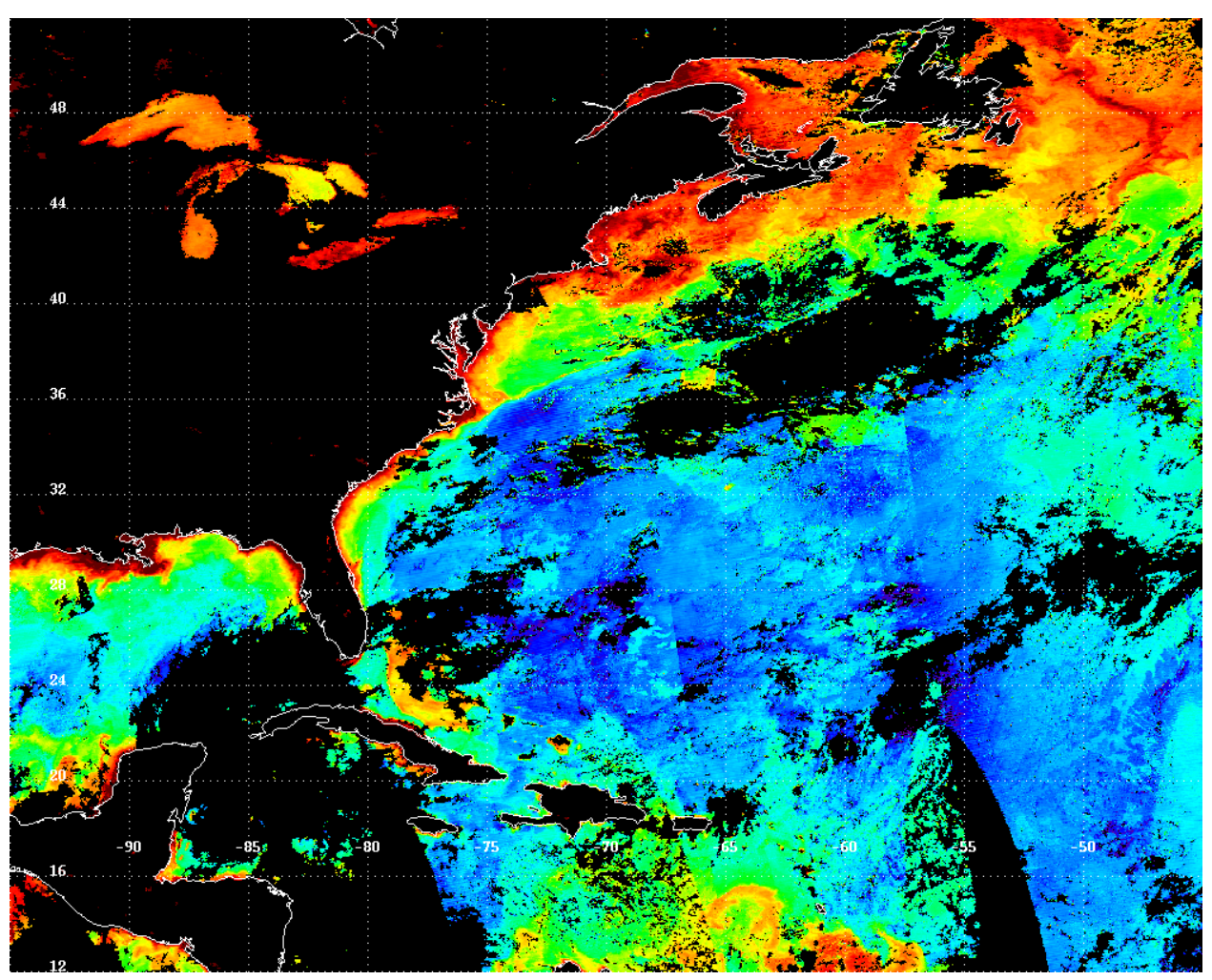

Figure 4. MODIS Aqua level-3 chlorophyll-a made from Aqua granules for Oct 12 and 132010 (days 285 and 286) in the area of the VIIRS granule coverage.

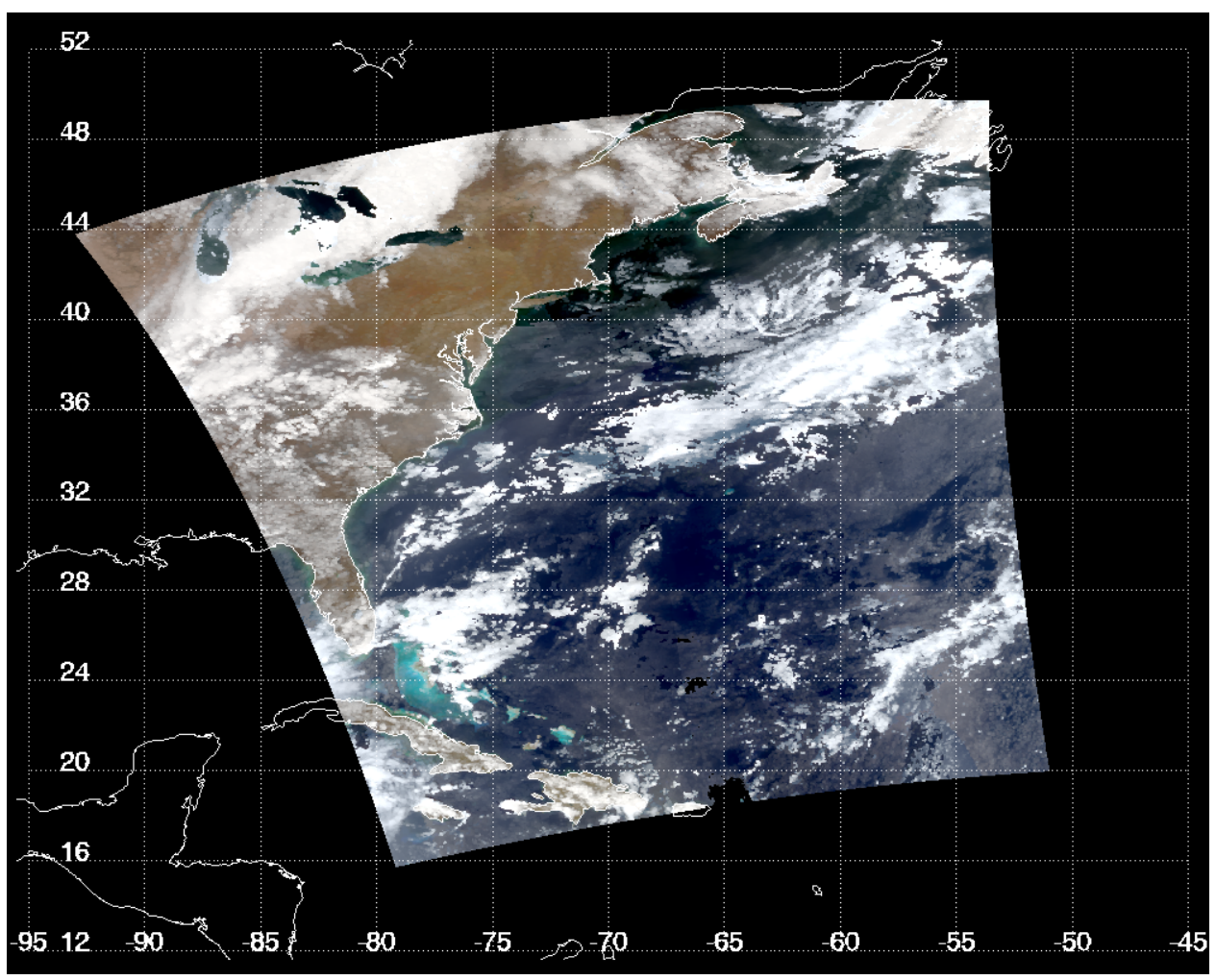

Figure 5. True color image made from the simulated VIIRS granules. 


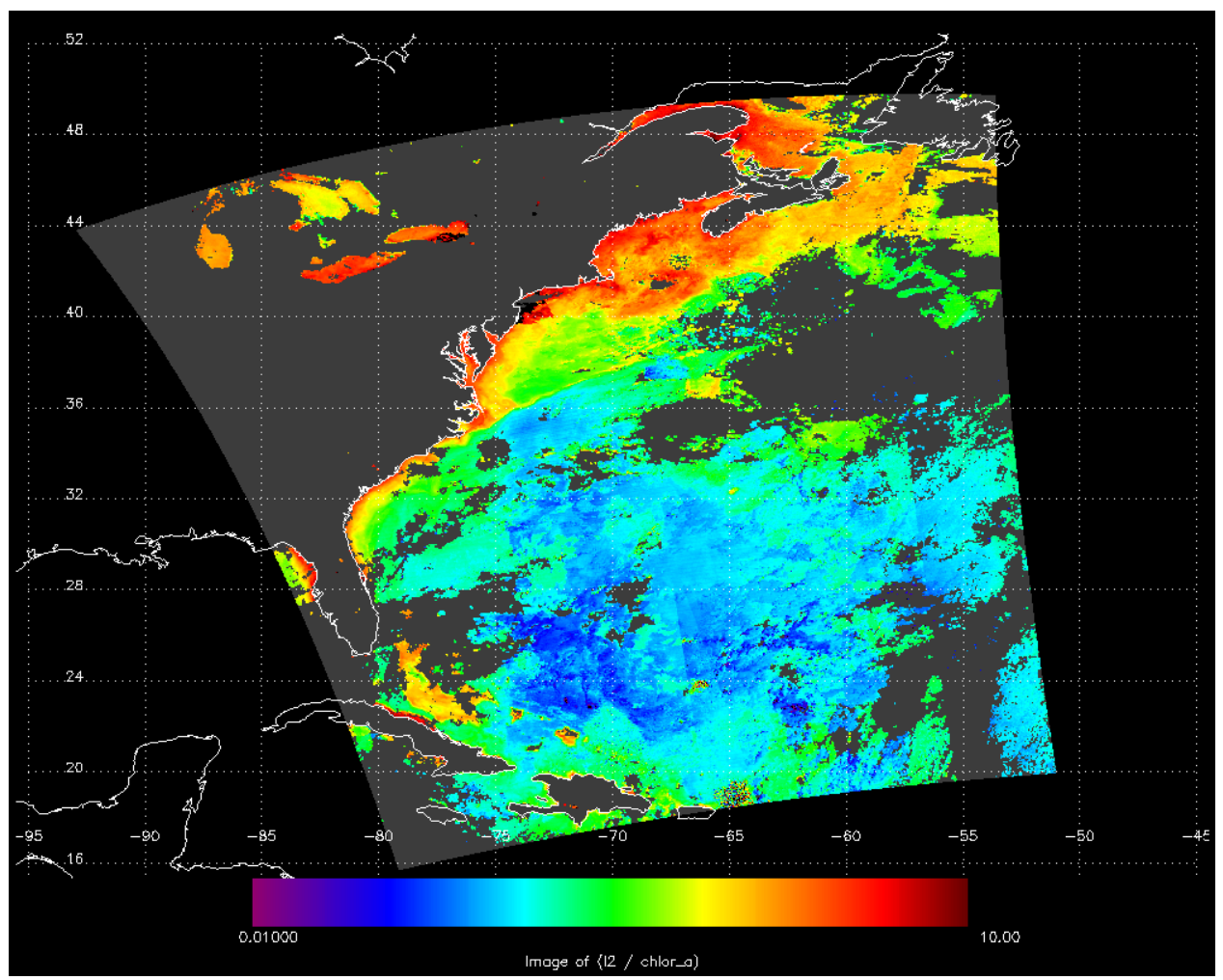

Figure 6. Chlorophyll-a image made from the simulated VIIRS granules processed to level-2.

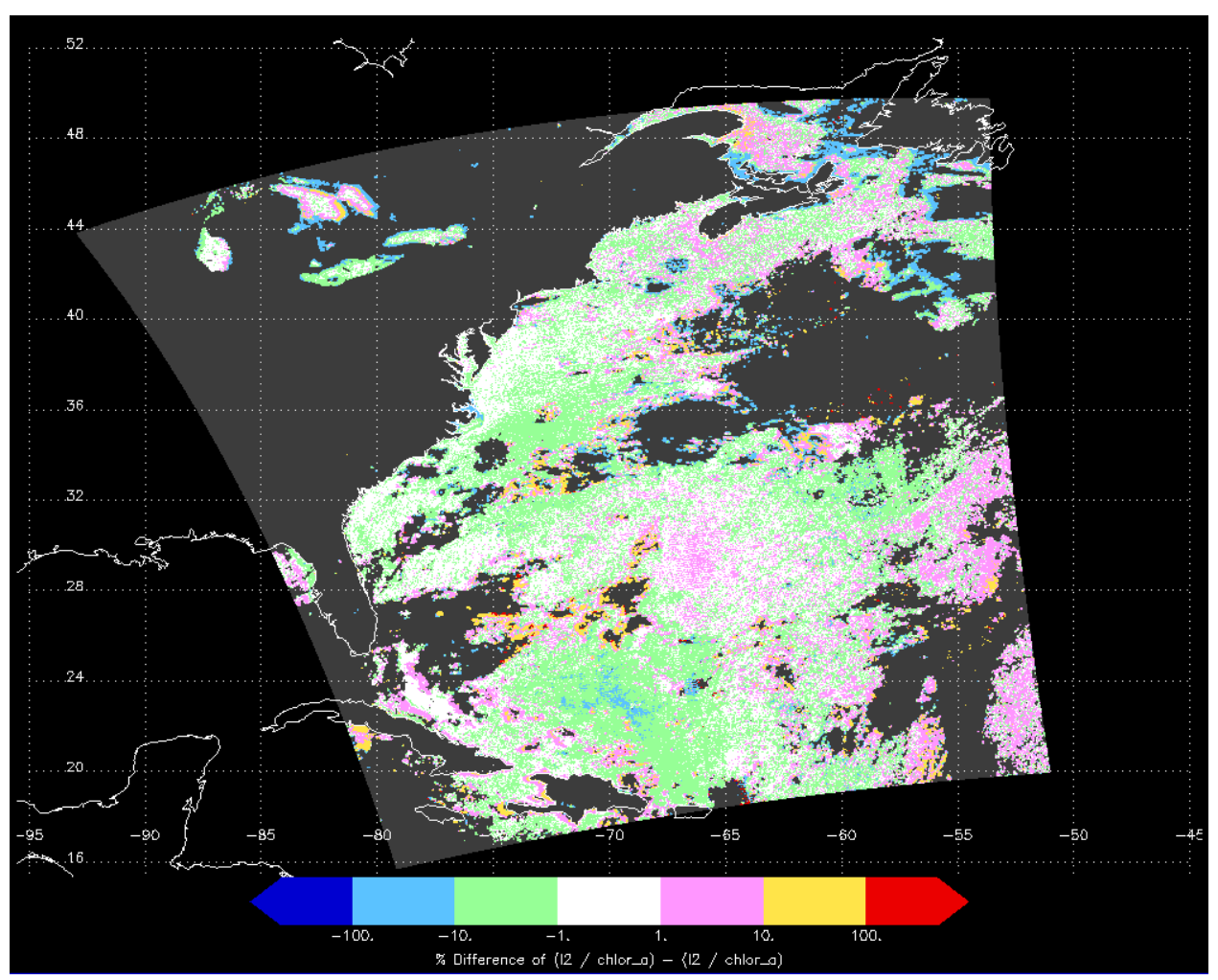

Figure 7. Percentage change in chlorophyll-a due to all 4 VIIRS artifacts. 


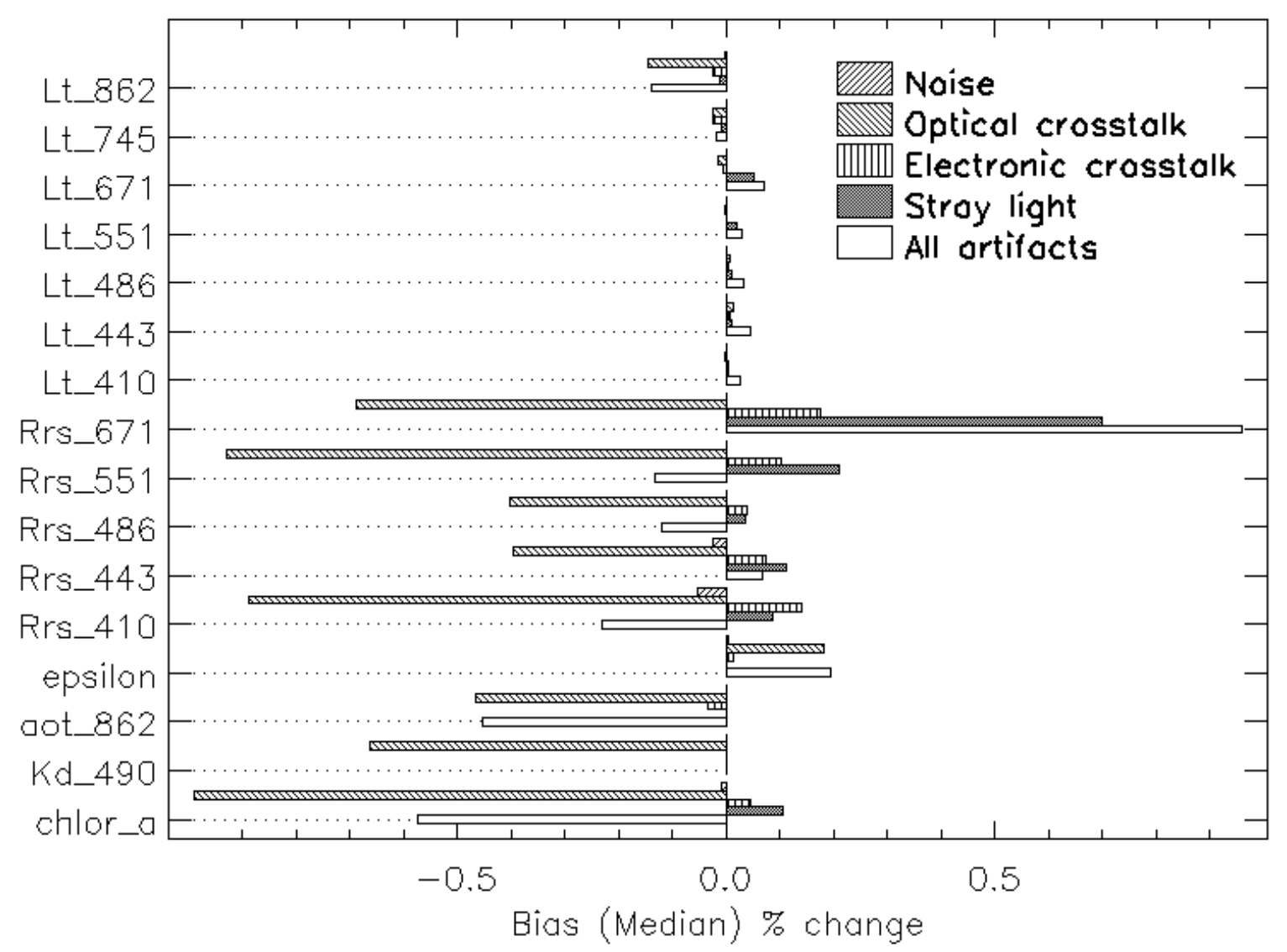

Figure 8. Bias (as a percentage of the data values) produced by four VIIRS artifacts and by the combination of the artifacts. Individual artifacts are noise, electronic and optical crosstalk, and stray light.

\section{INSTRUMENT ARTIFACT INFLUENCES}

The VIIRS simulator offers a unique opportunity to observe, measure, and possibly correct artifact influences in comparison to the same un-affected or ideal scene. Work is in progress to determine the effects of the artifacts. An initial set of results are discussed here.

The VIIRS imagery containing all the instrument artifacts shows only slight differences near some cloud and land edges. The true color (Fig 5) and chlorophyll (Fig 6) images show little apparent influence when compared to the ideal scene (not shown). An image of the amount of change in the chlorophyll is shown in Figure 7, and shows the percentage change over the 6 granule simulation example. Large changes of well over $10 \%$ and occasionally up to $100 \%$ are seen to occur in regions near some bright cloud or land features such as the North Atlantic, the Great Lakes, and in the Caribbean. Lesser amounts of change are seen around other clouds. Much of this is due to stray light effects but could also be due to optical and electrical crosstalk. The stray light mask for VIIRS will be developed using this kind of data to remove samples with unacceptable errors. Open ocean regions show changes of less than $10 \%$ - the source of those changes are currently unknown. The effects of noise show up throughout the scene as a general noise in the color coding of the image.

An initial evaluation was done of the size of the artifact influence on TOA radiances and derived ocean products throughout the example scene and illustrates the relative importance of the artifacts. The effect is measurable as both an overall bias from the ideal over the scene and also as an amount of RMS variation 


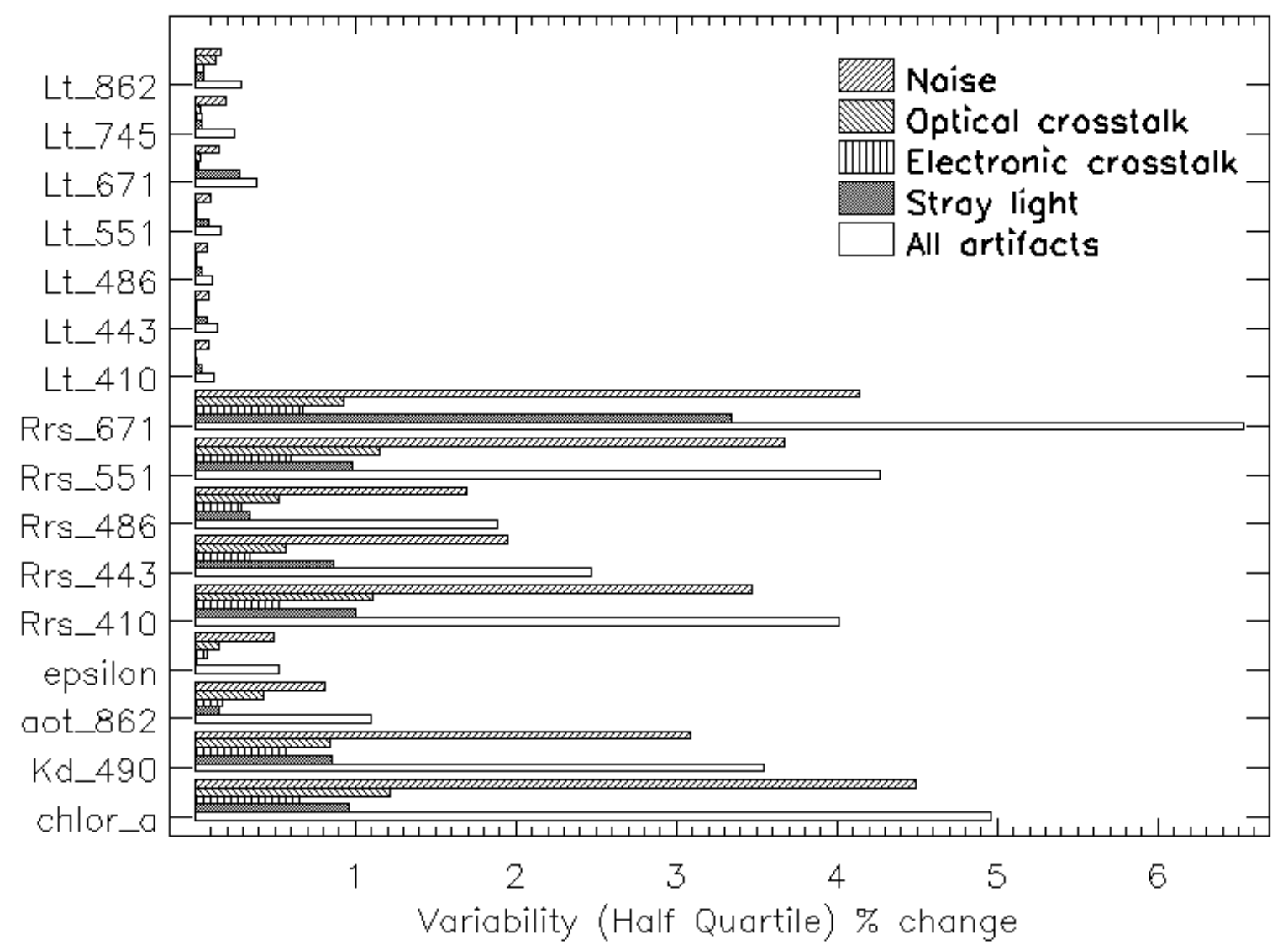

Figure 9. Variability (as a percentage of the data values) produced by each of four VIIRS artifacts and by the combination of the artifacts. The same artifacts are plotted here as in Figure 8

from the ideal. The plots in figures 8 and 9 show the effects of the artifact on bias and variation for selected parameters.

Figure 8 shows the bias induced by each of the 4 artifacts individually: noise, electronic and optical crosstalk, and stray light, as well as the cumulative effect of all 4 artifacts. The bias is reported for the TOA radiances: Lt_410 - 862, the water-leaving reflectance: Rrs_410 - 671, and in the derived parameters of chlorophyll: chlor_a, diffuse attenuation coefficient: Kd_490, aerosol optical thickness: aot_862, and epsilon, a measure of the aerosol type. Figure 9 shows the variability of the parameters for each artifact condition relative to the ideal scene. A comparison of the two figures shows that the bias is generally much smaller than the variability. Also, the bias will be naturally insensitive to the noise artifact, which should have an average bias of 0 and is the smallest effect in Fig 8. In contrast, the variability of the noise is one of the largest contributors, followed by optical crosstalk in bands M1, M3, and M4 and stray light in bands M2 and M5 Rrs. The overall effect is a 5\% variability in chlor_a, a 3.5\% variability in Kd_490 and about $1 \%$ in epsilon and aot_862. The greatest variability of $4 \%$ and over $6 \%$ occurs in the Rrs for M1 and M5. Fortunately, the 410 and 670 radiances are not as important in computation of chlorophyll. More specific statistical analysis over larger regions are planned for the next few months.

\section{SUMMARY}

The VIIRS SDR simulator has been improved to include the simulation of land and cloud radiances in addition to the ocean radiances. Many of the instrument artifacts are included and controllable in the 
simulator. Artifact impact testing is in the initial stages. Comparisons of the SDR radiances, derived waterleaving radiances, and products with and without instrument artifacts shows that variability is significantly larger than the bias. Instrument noise has the largest impact, followed by optical crosstalk and stray light influences. However, whereas the noise effects are distributed throughout the scene, the stray light, electronic and optical crosstalk effects are more pronounced in ocean regions near bright sources like land and clouds.

The general magnitude the artifact effect is less than $5 \%$ for most derived parameters.

\section{REFERENCES}

[1] Welsch, C., Swenson, H., Cota, S. A., DeLuccia, F., Haas, J. M., Schueler, C., Durham, R. M., Clement, J. E., and Ardanuy, P. E., "VIIRS (Visible Infrared Imager Radiometer Suite): A Next-Generation Operational Environmental Sensor for NPOESS," International Geoscience and Remote Sensing Symposium (IGARSS) Proceedings, 3, 1020-1022 (2001).

[2] Lee, T.F., Miller, S. D., Schueler, C., and Miller, S., NASA MODIS Previews NPOESS VIIRS Capabilities. Wea. Forecasting, 21, 649-655.

[3] Robinson,W.D., Patt, F. S., Franz, B. A., Turpie, K. R., and McClain, C. R., "A VIIRS Ocean Data Simulator", Proc. SPIE 7452, (2009).

[4] Patt, F. S., and W. W. Gregg, "Exact Closed-form Geolocation Algorithm for Earth Survey Sensors," Int. J. of Remote Sensing, 15, No. 18, (1994).

[5] [NPOESS Common Data Format Control Book - External, Vol. I - Overview, No. D34861-01, Rev. C], Northrop Grumman Space \& Mission Systems Corp. Space Technology, (2008).

[6] [NPOESS Common Data Format Control Book - External, Vol. III - SDT/TDR Formats, No. D34861-03, Rev. A], Northrop Grumman Space \& Mission Systems Corp. Space Technology, (2007).

[7] Franz, B.A., Werdell, P. J., Meister, G., Bailey, S. W., Eplee, R. E., Feldman, G. C., Kwiatkowska, E., McClain, C. R., Patt, F. S., Thomas, D., "The continuity of ocean color measurements from SeaWiFS to MODIS", Proc. SPIE 5882, (2005).

[8] Franz, B.A., Bailey, S. W., Werdell, P. J., and McClain, C. R., "Sensor-Independent Approach to Vicarious Calibration of Satellite Ocean Color Radiometry", Appl. Opt., 46 5068-5082 (2007).

[9] Franz, B. A., Ainsworth, E. J., Bailey, S., [In Situ Aerosol Optical Thickness Collected by the SIMBIOS Program (1997-2000): Protocols, and Data QC and Analysis], NASA/TM-2001-209982, Chapter 9 (2001).

[10] Meister, G. and McClain, C. R.. "Point-spread function of the ocean color bands of the Moderate Resolution Imaging Spectroradiometer on Aqua", Appl. Opt., 49, (2010).

[11] Waluschka, E. "Sensitivity of VIIRS polarization measurements", Proc. SPIE 7807, (2010)

[12] Barnes, R.A. and Butler, J. J., "Modeling spectral effects in Earth-observing satellite instruments", Proc. SPIE, 6744 (2007).

[13] Robinson, W. D., "The VIIRS inter-band filling technique", internal document (2010).

[14] "VIIRS Radiometric Calibration Algorithm Theoretical Basis Document ATBD (ref Y3261)", Northrop Grumman Space and Mission Systems Corp., (2007). 